\title{
An augmented Lagrangian multiplier method based on a CHKS smoothing function for solving nonlinear bilevel programming problems
}

\author{
Yan Jiang ${ }^{\mathrm{a}}$, Xuyong $\mathrm{Li}^{\mathrm{a}, *}$, Chongchao Huang ${ }^{\mathrm{b}}$, Xianing $\mathrm{Wu}^{\mathrm{c}}$ \\ ${ }^{a}$ State Key Laboratory of Urban and Regional Ecology, Research Center for Eco-Environmental Sciences, Chinese Academy of Sciences, Beijing 100085, PR China \\ ${ }^{\mathrm{b}}$ School of Mathematics and Statistics, Wuhan University, Wuhan 430072, PR China \\ ${ }^{\text {c }}$ Sinohydro Resources Limited, Beijing 100048, PR China
}

\section{A R T I C L E I N F O}

\section{Article history:}

Received 19 July 2012

Received in revised form 9 August 2013

Accepted 13 August 2013

Available online 23 August 2013

\section{Keywords:}

Nonlinear bilevel programming

Karush-Kuhn-Tucker condition

Complementary constraints

Smoothing function

Augmented Lagrangian multiplier method

\begin{abstract}
A B S T R A C T
Bilevel programming techniques deal with decision processes involving two decision makers with a hierarchical structure. In this paper, an augmented Lagrangian multiplier method is proposed to solve nonlinear bilevel programming (NBLP) problems. An NBLP problem is first transformed into a single level problem with complementary constraints by replacing the lower level problem with its Karush-KuhnTucker optimality condition, which is sequentially smoothed by a Chen-Harker-Kanzow-Smale (CHKS) smoothing function. An augmented Lagrangian multiplier method is then applied to solve the smoothed nonlinear program to obtain an approximate optimal solution of the NBLP problem. The asymptotic properties of the augmented Lagrangian multiplier method are analyzed and the condition for solution optimality is derived. Numerical results showing viability of the approach are reported.
\end{abstract}

() 2013 Elsevier B.V. All rights reserved.

\section{Introduction}

A bilevel programming (BLP) problem is characterized by a nested optimization problem with two levels, namely, an upper and a lower level, in a hierarchy where the constraint region of the upper level problem is implicitly determined by the lower level optimization problem. Such problems arise in game-theoretic and decomposition related applications in engineering design. Recently, these problems have received increasing attention and have been applied in various aspects of resource planning, management, and policy making, including water resource management, and financial, land-use, production, and transportation planning [1].

After a real-world problem has been modeled as a bilevel decision problem, how to find the optimal solution for this decision problem becomes very important. However, the nested optimization model described above is quite difficult to solve. It has been proved that solving a linear bilevel program is NP-hard [2,3]. In fact, even searching for a locally optimal solution of a linear bilevel program is strongly NP-hard [4]. Herminia and Carmen [5] pointed out that the existence of multiple optima for the lower-level problem can result in an inadequate formulation of the bilevel problem. Moreover, the feasible region of the problem is usually

\footnotetext{
* Corresponding author. Tel.: +86 1062840210.

E-mail address: yanjiang@rcees.ac.cn (X.Y. Li).
}

non-convex, without necessarily being a connected set, and empty, making it difficult to solve BLP problems.

Existing methods for solving BLP problems can be classified into the following categories [6]: extreme-point search, transformation, descent and heuristic, interior point, and intelligent computation approaches. Recently, the latter approach has become an important computational method for solving BLP problems. Genetic algorithm [7-9], Neural networks [10], Tabu search [11-13], ant colony optimization [14], and particle swarm optimization [15-17] are typical intelligent algorithms for solving BLP problems. In all these algorithms, the BLP problem is first reduced to a one-level problem with complementary constraints by replacing the lower level problem with its Karush-Kuhn-Tucker (KKT) optimality condition. However, the one-level complementary slackness problem is non-convex and non-differential. The major difficulty in solving this type of problem is that its constraints fail to satisfy the standard Mangasarian-Fromovitz constraint qualification at any feasible point, and that general intelligent algorithms may fail to uncover such local minima or even mere stationary points.

To avoid the difficulty of the nonsmooth constraints, a sequence of smooth problems by using smoothing functions have been progressively approximated this nonsmooth problem. To date, there have been many smoothing functions, such as perturbed Fischer-Burmeister (FB), Chen-Harker-Kanzow-Smale (CHKS), Neural networks, uniform, and Picard smoothing functions [18-20]. Thereinto, perturbed FB and CHKS smoothing functions 
have been applied widely to solve BLP problems. Facchinei et al. [21] transformed mathematical program with equilibrium constraints (MPEC), including bilevel programming problems as a particular case, into an equivalent one-level nonsmooth optimization problem, which was then approximated progressively by applying CHKS smoothing function to a sequence of smooth, regular problems that approximate the nonsmooth problem. Finally, it was solved by standard available software for constrained optimization. Dempe [22] introduced a smoothing technique by applying perturbed FB function and Lagrangian function method to solve a BLP problem, approximated by a sequence of smooth optimization problems. Wang et al. [23] proposed an approximate programming method based on the simplex method to research the general bilevel nonlinear programming model by applying perturbed FB smoothing function. Etoa [24] presented a smoothing sequential quadratic programming using perturbed FB functional to compute a solution of a quadratic convex bilevel programming problem. In general, perturbed FB and CHSK smoothing functions are very closely related. These two functions are very similar except for the difference of a constant. In what follows, we choose to focus on the CHKS smoothing function to approximate nonsmooth problem.

The purpose of this work is to design an efficient algorithm for solving general nonlinear bilevel programming (NBLP) models. Different from the algorithms in [22,23], where Lagrangian optimization and simplex method were used to solve a smooth problem approximated by perturbed FB smoothing function, we apply a CHKS smoothing function and an augmented Lagrangian multiplier method to solve NBLP problem, and focus on the detailed analysis on the condition for solution optimality. The remainder of this paper is organized as follows: Section 2 presents the formulation and basic definitions of bilevel programming, while Section 3 introduces the smoothing method for nonlinear complementarity problems. In Section 4 we explain the augmented Lagrangian multiplier method in detail and derive the condition for asymptotic stability, solution feasibility, and solution optimality. Numerical examples are reported in Section 5 and Section 6 concludes the paper.

\section{Formulation of an NBLP problem}

The general formulation of NBLP problems is as follows [25]:

$$
\begin{array}{ll}
\min _{x} & F(x, y), \\
\text { s.t. } & G(x, y) \leqslant 0, \\
& \min _{y} f(x, y), \\
& \text { s.t. } \quad g(x, y) \leqslant 0, \\
& \quad x \in X \subset R^{n_{1}}, \quad y \in Y \subset R^{n_{2},}
\end{array}
$$

where $F, f: R^{n_{1}} \times R^{n_{2}} \rightarrow R$ represent the objective function of the leader and the follower, respectively, $G: R^{n_{1}} \times R^{n_{2}} \rightarrow R^{m_{1}}$ denotes the constraints of the leader, $g: R^{n_{1}} \times R^{n_{2}} \rightarrow R^{m_{2}}$ denotes the constraints of the follower, and $x \in R^{n_{1}}, y \in R^{n_{2}}$ denote the decision variables of the leader and the follower, respectively. The following definitions are associated with the problem (1). The relaxed feasible set (or constrained region) is defined as $S=\{(x, y): G(x, y) \leqslant 0, g(x$, $y) \leqslant 0\}$, the lower level feasible set for each fixed $x$ as $S(x)=\{y$ : $g(x, y) \leqslant 0\}$, the follower's rational reaction set as $P(x)=\{y: y \in \arg \min \{f(x, \hat{y}), \hat{y} \in S(x)\}\}$, and the inducible region as $I R=\{(x, y):(x, y) \in S, y \in P(x)\}$.

To guarantee that there is at least one solution to the NBLP problem, we assume that the constrained region $S$ is nonempty and compact. At the same time, for some fixed $x \in X$, we assume that the lower level problem is a convex optimization problem satisfying the Mangasarian-Fromovitz constraints qualification (MFCQ) for any $y \in S(x)$. Then we can reduce the bilevel programming problem to a one-level problem using the KKT condition.

$$
\begin{array}{cl}
\min _{x, y, \lambda} & F(x, y), \\
\text { s.t. } & G(x, y) \leqslant 0, \\
& \nabla_{y} L(x, y, \lambda)=0, \\
& \lambda^{T} g(x, y)=0, \\
& g(x, y) \leqslant 0, \\
& \lambda \geqslant 0,
\end{array}
$$

where $L(x, y, \lambda)=f(x, y)+\lambda^{T} g(x, y), \lambda \in R^{m_{2}}$ is a Lagrange function.

\section{Smoothing method}

Problem (2) is a mathematical program with nonlinear complementary constraints. It is non-convex, non-differentiable and the regularity assumptions, which are needed to handle smooth optimization programs successfully, are never satisfied. So it is not appropriate to use standard nonlinear programming software to solve it [26,27].

We consider the following perturbation of problem (2):

$$
\begin{aligned}
\min _{x, y, \lambda} & F(x, y), \\
\text { s.t. } & G(x, y) \leqslant 0, \\
& \nabla_{y} L(x, y, \lambda)=0, \\
& \lambda^{T} g(x, y)=\epsilon^{2}, \\
& g(x, y) \leqslant 0, \\
& \lambda \geqslant 0,
\end{aligned}
$$

where $\epsilon>0$ is an identity element. For a given $\epsilon$, we solve problem (3) to get the solution $\left(x_{\epsilon}, y_{\epsilon}, \lambda_{\epsilon}\right)$. As $\epsilon$ decreases slowly toward 0 , the optimum of $F_{\epsilon}$ follows to the global optimum of $F_{0}$. This set of constraint conditions is called the central path conditions as they define a trajectory approaching the solution set for $\epsilon \rightarrow 0$. The smoothing method is an effective way to deal with this issue. The main feature of smoothing method is to reformulate the problem (3) as a smooth nonlinear programming problem by replacing the difficult equilibrium constraint with a smoothing function. Consequently, the true problem (3) can be approximated by a sequence of smooth problem $[21,28,29]$. The numerical evidence on the efficiency of the smoothing approach is very impressive. Hence, In this work, we smooth the nonlinear programming with complementary constraints by applying CHKS smoothing function.

Define function $\phi_{\epsilon}: R^{3} \rightarrow R$ using the CHKS smoothing function $\phi_{\epsilon}(a, b)=a+b-\sqrt{(a-b)^{2}+4 \epsilon^{2}}$. Then, we can have the following proposition [21].

Proposition 1. For every $\epsilon \in R$, we have $\phi_{\epsilon}(a, b)=0$, if and only if $a \geqslant 0, b \geqslant 0, a b=\epsilon^{2}$ for $\epsilon \geqslant 0$, where $\epsilon$ is a parameter converging to zero.

Note that for every $\epsilon \geqslant 0, \phi_{\epsilon}(a, b)$ is continuously differentiable with respect to $(a, b)$. By applying the CHKS smoothing function $\phi_{\epsilon}(x, y)$, problem (3) can be approximated by:

$$
\begin{aligned}
\min _{x, y, \lambda} & F(x, y), \\
\text { s.t. } & G(x, y) \leqslant 0 \\
& \nabla_{y} L(x, y, \lambda)=0 \\
& \lambda_{j}-g_{j}(x, y)-\sqrt{\left(\lambda_{i}+g_{j}(x, y)\right)^{2}+4 \epsilon^{2}}=0, j=1,2, \ldots, m_{2} .
\end{aligned}
$$

Problem (4) can be viewed as a perturbation of problem (2). Problem (4) overcomes the difficulty due to problem (2) not satisfying any regularity assumption and paves the way for using an augmented Lagrangian multiplier approach to solve problem (2). 
The idea of the algorithm is as followed: the smoothing method starts with a large $\epsilon$, finds the optimum of $F_{\epsilon}$, decreases $\epsilon$ gradually, and recomputes the optimum at each step-starting from the previous solution-until $\epsilon$ is small enough. We claim that this procedure yields near-optimal solutions.

To simply our discussion and for convenience, we introduce the following notations: Let $h(x, y, \lambda)=G(x, y), \quad H(x, y, \lambda)$ $=\left[\begin{array}{c}\nabla_{y} L(x, y, \lambda) \\ \phi_{\epsilon}\left(\lambda_{j},-g(x, y)\right)_{j=1,2 \ldots, m_{2}}\end{array}\right]$ and $z=(x, y, \lambda)$, then problem (4) can be written as:

$$
\begin{array}{cl}
\min _{z} & F(z), \\
\text { s.t. } & h(z) \leqslant 0, \\
& H(z)=0 .
\end{array}
$$

Definition 1. Let $z$ be a feasible point of problem (4). We say that $z$ is a regular point of problem (5) if gradients $\nabla_{z} h(z)$ and $\nabla_{z} H(z)$ are linearly independent.

Similar to the main result, Theorem 3, in [21], the theorem below follows directly.

Theorem 1. Let $\left\{z^{\epsilon}\right\}$ be a sequence of solutions of problem (5). If sequence $\left\{z^{\epsilon}\right\}$ is contained in a compact set and converges to some $\bar{z}$ for $\epsilon \rightarrow 0$, then $\bar{z}$ is a global solution of problem (2).

\section{Augmented Lagrangian multiplier method}

\subsection{Definition of augmented Lagrangian multiplier method}

During the past few decades, considerable attention has been focused on finding methods for solving nonlinear programming problem (5) using unconstrained minimization techniques. Usually, a penalty function method, which requires that an infinite sequence of penalty problems be solved, is used to solve this type of problem. The main drawback of the sequential penalty function method is that when the penalty parameter approaches its limiting value, the corresponding penalty problems are ill-conditioned, resulting in various shortcomings such as numerical instability and slow convergence. At the same time, owing to the presence of non-convexity in the smooth approximate problems (5), multiple local optimal solutions may exist and thus, the traditional Lagrangian method is not guaranteed to find a global solution of the approximation problem. To overcome these shortcomings, we apply an augmented Lagrangian multiplier algorithm [30,31], which combines the Lagrangian multiplier method and penalty method, to solve nonlinear programming problems. Numerical studies indicate that the augmented Lagrangian multiplier method offers significant advantages over the usual penalty methods. The augmented Lagrangian function is defined as:

$$
\begin{aligned}
\Phi(z, w, v, \sigma)= & F(z)+\frac{1}{2 \sigma} \sum_{j=1}^{m_{1}}\left\{\left[\max \left(0, w_{j}+\sigma h_{j}(z)\right)\right]^{2}-w_{j}^{2}\right\} \\
& +\sum_{j=1}^{m_{2}+n_{2}} v_{j} H_{j}(z)+\frac{\sigma}{2} \sum_{j=1}^{m_{2}+n_{2}} H_{j}(z)^{2},
\end{aligned}
$$

where $w \in R^{m_{1}}$, and $v \in R^{n_{2}+m_{2}}$ are multipliers corresponding to the inequality and equality, separately, and $\sigma$ is a positive penalty factor.

The augmented Lagrangian multiplier method for solving nonlinear programming problems can be stated as follows. Suppose that $w^{(k)}$ and $v^{(k)}$ are Lagrangian multiplier vectors and $z^{(k)}$ is the minimum of $\Phi\left(z, w^{(k)}, v^{(k)}, \sigma\right)$ at iteration $k$. Then, the update formulas for multiplier $w^{(k+1)}$ and $v^{(k+1)}$ at iteration $k+1$ are given as:

$$
\begin{aligned}
& w^{(k+1)}=\max \left(0, w^{(k)}+\sigma h\left(z^{(k)}\right)\right), \\
& v^{(k+1)}=v^{(k)}-\sigma H\left(z^{(k)}\right) .
\end{aligned}
$$

Denoting the solution by $z^{(k+1)}$, we solve the unconstrained program $\min \Phi(z, w, v, \sigma)$. After a certain number of iterations, if $w^{(k)} \rightarrow \bar{w}$ and $v^{(k)} \rightarrow \bar{v}$, then $z^{(k)} \rightarrow \bar{z}$. If $\left\{w^{(k)}\right\}$ and $\left\{v^{(k)}\right\}$ do not converge or converge very slowly, we increase the value of penalty parameter $\sigma$ and repeat the above steps.

The steps of the augmented Lagrangian multiplier algorithm for NBLP problems are given as follows:

Step 1 (Initialization). Choose initial value $z^{(0)}$, Lagrangian multiplier factors $w^{(1)}, v^{(1)}$ and penalty parameter $\sigma$. Give expansion coefficient $\alpha>1$, reduced coefficient $\beta \in(0,1)$ and allowed error $\varepsilon>0$. Set $k=1$.

Step 2 (Solution of the problem (7)). Solve the unconstrained programming problem $\min \Phi\left(z^{(k-1)}, w^{(k)}, v^{(k)}, \sigma\right)$ and obtain the optimal solution $z^{(k)}$.

Step 3 (Termination check). If $\left\|h\left(z^{(k)}\right)<\varepsilon\right\|$ and $\left\|H\left(z^{(k)}\right)<\varepsilon\right\|$, then the algorithm terminates. $z^{(k)}$ is taken as the approximate optimal solution and $F\left(z^{(k)}\right)$ is the approximate optimal value. Otherwise, go to Step 4.

Step 4 (Updating penalty parameter). If $\frac{\left\|h\left(z^{(k)}\right)\right\|}{\left\|h\left(z^{(k-1)}\right)\right\|} \geqslant \beta$ or $\frac{\left\|H\left(z^{(k)}\right)\right\|}{\left\|H\left(z^{k-1}\right)\right\|} \geqslant \beta$, let $\sigma=\alpha \sigma$, and go to Step $\mathbf{5}$.

Step 5 (Updating multipliers). Update the multipliers using Eqs. (7) and (8). Let $k=k+1$ and go to Step 2.

The relevant feature of the augmented Lagrangian function method is that, given suitable assumptions, the solution of the constrained optimization problem can be obtained by recursively updating the multiplier vector, without increasing penalty parameter $\sigma$ to infinity.

\subsection{Optimality results}

Next we study the relationship between the solution of problem (5) and the unconstrained minimum points of $\Phi(z, w, v, \sigma)$.

If we use min instead of max, we have:

$$
\begin{aligned}
\Phi(z, w, v, \sigma)= & F(z)+\sum_{j=1}^{m_{1}} w_{j} h_{j}(z)+\frac{\sigma}{2} \sum_{j=1}^{m_{1}} h_{j}(z)^{2} \\
& -\frac{1}{2 \sigma} \sum_{j=1}^{m_{1}}\left[\min \left(0, h_{j}(z)+\frac{w_{j}}{\sigma}\right)\right]^{2}+\sum_{j=1}^{m_{2}+n_{2}} v_{j} H_{j}(z) \\
& +\frac{\sigma}{2} \sum_{j=1}^{m_{2}+n_{2}} H_{j}(z)^{2} .
\end{aligned}
$$

Define $d_{i}^{2}(z, w, \sigma)=-\min \left\{0, h_{i}(z)+\frac{w_{i}}{\sigma}\right\}, i=1, \ldots, m_{1}$ and denote $d(z, w, \sigma)=\left(d_{1}(z, w, \sigma), \ldots, d_{m_{1}}(z, w, \sigma)\right)^{T}, \quad$ and $D(z, \quad w$, $\sigma)=\operatorname{diag}\left(d_{i}(z, w, \sigma)\right)$. It can easily be verified that:

$$
\begin{aligned}
\Phi(z, w, v, \sigma)= & F(z)+w^{T}[h(z)+D(z, w, \sigma) d(z, w, \sigma)]+\frac{\sigma}{2} \| h(z) \\
& +D(z, w, \sigma) d(z, w, \sigma)\left\|^{2}+v^{T} H(z)+\frac{\sigma}{2}\right\| H(z) \|^{2} .
\end{aligned}
$$

The Lagrangian function $L^{\prime}$ for problem (5) is defined as:

$L^{\prime}(z, w, v)=F(z)+w^{T} h(z)+v^{T} H(z)$.

Under the assumption that $F, h$ and $H$ are continuously differentiable on $R^{n_{1}+n_{2}}$, we obtain the following results.

Theorem 2. For any $\sigma>0$, let $(\bar{z}, \bar{w}, \bar{v})$ be a stationary point of $\Phi(z, w$, $v, \sigma)$. Then, $(\bar{z}, \bar{w}, \bar{v})$ is a KKT pair for problem (5) and $F(\bar{z})=\Phi(\bar{z}, \bar{w}, \bar{v}, \sigma)$. 
Proof. We have the following expressions for the components of the gradient of $\Phi(z, w, v, \sigma)$ :

$$
\begin{aligned}
\nabla_{z} \Phi(z, w, v, \sigma)= & \nabla_{z} L^{\prime}(z, w, v)+2 \sigma \frac{\partial h(z)^{T}}{\partial z}[h(z) \\
& +D(z, w, \sigma) d(z, w, \sigma)]+2 \sigma \frac{\partial H(z)^{T}}{\partial z} H(z),
\end{aligned}
$$

$\nabla_{w} \Phi(z, w, v, \sigma)=h(z)+D(z, w, \sigma) d(z, w, \sigma)$,

$\nabla_{v} \Phi(z, w, v, \sigma)=H(z)$

If $(\bar{z}, \bar{w}, \bar{v})$ is a stationary point of $\Phi(z, w, v, \sigma)$, then $\nabla_{z} \Phi(\bar{z}, \bar{w}, \bar{v}, \sigma)=0, \nabla_{w} \Phi(\bar{z}, \bar{w}, \bar{v}, \sigma)=0, \quad$ and $\quad \nabla_{\nu} \Phi(\bar{z}, \bar{w}, \bar{v}, \sigma)=0$. This implies that $\nabla_{z} L^{\prime}(\bar{z}, \bar{w}, \bar{v})=0, h(\bar{z})+D(\bar{z}, \bar{w}, \sigma) d(z, w, \sigma)=0$, and $H(\bar{z})=0$. Then we have:

$$
\begin{aligned}
h_{i}(\bar{z})+d_{i}^{2}(\bar{z}, \bar{w}, \sigma) & =h_{i}(\bar{z})-\min \left[0, h_{i}(\bar{z})+\frac{w_{i}}{\sigma}\right] \\
& =\max \left[h_{i}(\bar{z}),-\frac{w_{i}}{\sigma}\right]=0 .
\end{aligned}
$$

This implies that $h_{i}(\bar{z}) \leqslant 0$ and $w^{T} h(\bar{z})=0$, which shows that $(\bar{z}, \bar{w}, \bar{v})$ is a KKT pair for problem (5). By substituting $h(z)+D(\bar{z}, \bar{w}, \sigma) d(z, w, \sigma)=0$ and $H(\bar{z})=0$ into (10), we get $F(\bar{z})=\Phi(\bar{z}, \bar{w}, \bar{v}, \sigma)$. This completes the proof.

Theorem 3. Let $(\bar{z}, \bar{w}, \bar{v})$ be an unconstrained global minimum point of $\Phi(z, \quad w, v, \quad \sigma)$ of $\epsilon \rightarrow 0$ on subset $Z \times W \times V \subseteq R^{n_{1}+n_{2}+m_{2}}$ $\times R^{m_{1}} \times R^{m_{2}+n_{2}}$. Then, $(\bar{z}, \bar{w}, \bar{v})$ is a KKT pair for problem (5), and $\bar{z}$ is a global minimum point of problem (5) and also a global minimum point of the NBLP problem.

Proof. The fact that $(\bar{z}, \bar{w}, \bar{v})$ is an unconstrained global minimum point of $\Phi(z, w, v, \sigma)$ implies that $\bar{z}$ is an unconstrained global minimum point of $\Phi(z, \bar{w}, \bar{v}, \sigma), \nabla_{z} \Phi(\bar{z}, \bar{w}, \bar{v}, \sigma)=0, \nabla_{w} \Phi(\bar{z}, \bar{w}, \bar{v}, \sigma)=0$, and $\nabla_{v} \Phi(\bar{z}, \bar{w}, \bar{v}, \sigma)=0$. Therefore, $(\bar{z}, \bar{w}, \bar{v})$ is a stationary point of $\Phi(z, w, v, \sigma)$. And by Theorem $2,(\bar{z}, \bar{w}, \bar{v})$ is a KKT pair for problem (5) and $F(\bar{z})=\Phi(\bar{z}, \bar{w}, \bar{v}, \sigma)$.

Since $\bar{z}$ is an unconstrained global minimum point of $\Phi(z, \bar{w}, \bar{v}, \sigma)$ on subset $Z \times W \times V$, there exists a neighborhood $\Omega$ of $\bar{z}$, such that

$F(\bar{z}) \leqslant \Phi(z, \bar{w}, \bar{v}, \sigma), \quad \forall z \in \Omega$

By Eq. (9), the above inequality yields:

$F(\bar{z}) \leqslant F(z), \quad \forall z \in \Omega \bigcap\{z: h(z) \leqslant 0, H(z)=0\}$.

So, $\bar{z}$ is a global minimum point of problem (5). From Theorem 1 above, we can complete the proof.

\section{Numerical experiments}

In this section, we present three examples to illustrate the feasibility of the augmented Lagrangian multiplier algorithm for NBLP problems.

Example 1 [32]. Consider the following NBLP problem, where $x \in R^{2}, y \in R^{2}$.

$$
\begin{array}{ll}
\min _{x \geqslant 0} & F(x, y)=-x_{1}^{2}-3 x_{2}-4 y_{1}+y_{2}^{2}, \\
\text { s.t. } & x_{1}^{2}+2 x_{2} \leqslant 4, \\
& \min _{y \geqslant 0} f(x, y)=2 x_{1}^{2}+y_{1}^{2}+x_{2}^{2}+y_{1}^{2}-5 y_{2}, \\
& \text { s.t. } \quad x_{1}^{2}-2 x_{1}+x_{2}^{2}-2 y_{1}+y_{2} \geqslant-3, \\
& x_{2}+3 y_{1}-4 y_{2} \geqslant 4 .
\end{array}
$$

For mixed $x$, the lower level problem satisfies MFCQ. Applying the KKT condition, problem (10) can be transformed into the following single-level programming problem:

$$
\begin{array}{rl}
\min _{x, y, \lambda \geqslant 0} & F(x, y)=-x_{1}^{2}-3 x_{2}-4 y_{1}+y_{2}^{2}, \\
\text { s.t. } & x_{1}^{2}+2 x_{2} \leqslant 4 \\
& 2 y_{1}+2 \lambda_{1}-3 \lambda_{2}=0 \\
& -5-\lambda_{1}+4 \lambda_{2}=0 \\
& \lambda_{1}\left(x_{1}^{2}-2 x_{1}+x_{2}^{2}-2 y_{1}+y_{2}+3\right)=0, \\
& \lambda_{2}\left(x_{2}+3 y_{1}-4 y_{2}-4\right)=0 \\
& x_{1}^{2}-2 x_{1}+x_{2}^{2}-2 y_{1}+y_{2} \geqslant-3 \\
& x_{2}+3 y_{1}-4 y_{2} \geqslant 4
\end{array}
$$

Adopting the CHSK smoothing function, $\phi_{\epsilon}(a, b)=a+b-\sqrt{(a-b)^{2}+4 \epsilon^{2}}$, problem (11) can be approximated by the following problem:

$$
\begin{array}{rl}
\min _{x y, \lambda \geqslant 0} & F(x, y)=-x_{1}^{2}-3 x_{2}-4 y_{1}+y_{2}^{2}, \\
\text { s.t. } \quad & x_{1}^{2}+2 x_{2} \leqslant 4, \\
& 2 y_{1}+2 \lambda_{1}-3 \lambda_{2}=0, \\
& -5-\lambda_{1}+4 \lambda_{2}=0, \\
& \lambda_{1}+\left(x_{1}^{2}-2 x_{1}+x_{2}^{2}-2 y_{1}+y_{2}+3\right)- \\
& \sqrt{\left(\lambda_{1}-\left(x_{1}^{2}-2 x_{1}+x_{2}^{2}-2 y_{1}+y_{2}+3\right)\right)^{2}+4 \epsilon^{2}}=0, \\
& \lambda_{2}+\left(x_{2}+3 y_{1}-4 y_{2}-4\right)- \\
& \sqrt{\left(\lambda_{2}-\left(x_{2}+3 y_{1}-4 y_{2}-4\right)\right)^{2}+4 \epsilon^{2}}=0 .
\end{array}
$$

Let $z=\left(x_{1}, x_{2}, y_{1}, y_{2}, \lambda_{1}, \lambda_{2}\right)^{T}$. Set the initial point $z^{(0)}=(1,1,1,1,1,1)^{T}, \quad \sigma=100, \quad w^{(0)}=0.5, \quad v^{(0)}=(0.5,0.5,0.5,0.5)^{T}$, $\alpha=10, \beta=0.25$, and $\varepsilon=0.001$. According to the above steps, the results listed in Table 1 are obtained for different values of $\epsilon$.

Example 2 [33]. Consider the following NBLP problem, where $x \in R^{1}, y \in R^{1}$.

$$
\begin{array}{rl}
\min _{x \geqslant 0} & F(x, y)=x^{2}+(y-10)^{2}, \\
\text { s.t. } & 0 \leqslant x \leqslant 15, \\
& \min _{y \geqslant 0} \quad f(x, y)=(x+2 y-30)^{2}, \\
& \text { s.t. } \quad x-y^{2} \geqslant 0, \\
& 20-x-y^{2} \geqslant 0 .
\end{array}
$$

After applying the KKT condition and CHKS smoothing function, the above problem can be transformed into a problem similar to problem (5) which can be solved by using the augmented Lagrangian multiplier method. Let $z=\left(x, y, \lambda_{1}, \lambda_{2}\right)^{T}$ and set the initial point $z^{(0)}=(1,1,1,1)^{T}$. Parameters $\sigma, w^{(0)}, v^{(0)}, \alpha, \beta$, and $\varepsilon$ are selected as in Example 1. Table 1 presents the different optimal solutions of Example 2 for varying small values of $\epsilon$.

Example 3 [34]. Consider the following nonlinear BLP problem, where $x \in R^{1}, y \in R^{2}$.

$$
\begin{array}{rl}
\min _{x \geqslant 0} & F(x, y)=(x-1)^{2}+2 y_{1}^{2}-2 x, \\
& \min _{y \geqslant 0} \quad f(x, y)=\left(2 y_{1}-4\right)^{2}+\left(2 y_{2}-1\right)^{2}+x y_{1}, \\
\text { s.t. } \quad & 4 x+5 y_{1}+4 y_{2} \leqslant 12, \\
& -4 x-5 y_{1}+4 y_{2} \leqslant-4, \\
& 4 x-4 y_{1}+5 y_{2} \leqslant 4, \\
& -4 x+4 y_{1}+5 y_{2} \leqslant 4 .
\end{array}
$$


Table 1

Comparision of the solutions in our paper with those of references.

\begin{tabular}{|c|c|c|c|c|c|c|c|}
\hline \multirow[t]{2}{*}{$P$} & \multirow[t]{2}{*}{$\epsilon$} & \multicolumn{2}{|l|}{ CHKS } & \multicolumn{2}{|l|}{ PFB } & \multicolumn{2}{|c|}{ results in the references } \\
\hline & & $(x, y)^{\mathrm{a}}$ & $F(x, y)^{b}$ & $(x, y)^{\mathrm{a}}$ & $F(x, y)^{\mathrm{b}}$ & $(x, y)^{\mathrm{a}}$ & $F(x, y)^{\mathrm{b}}$ \\
\hline \multirow[t]{3}{*}{1} & 0.1 & $(0,2,1.997,0.969)$ & -12.969 & $(0,2,1.933,0.872)$ & -12.972 & & \\
\hline & 0.01 & $(0,2,1.990,0.982)$ & -12.996 & $(0,2,1.944,0.891)$ & -12.982 & $(0,2,1.875,0.906)$ & -12.679 \\
\hline & 0.001 & $(0,2,1.997,0.995)$ & -12.998 & $(0,2,1.949,0.898)$ & -12.990 & & \\
\hline \multirow[t]{3}{*}{2} & 0.1 & $(2.6043,1.6107)$ & 77.1630 & $(2.6000,1.6120)$ & 77.1190 & & \\
\hline & 0.01 & $(2.6009,1.6124)$ & 77.1162 & $(2.5992,1.6119)$ & 77.1161 & $(2.600,1.612)$ & 77.119 \\
\hline & 0.001 & $(2.6006,1.6126)$ & 77.1115 & $(2.5992,1.6122)$ & 77.1110 & & \\
\hline \multirow[t]{3}{*}{3} & 0.1 & $(1.310,0.342,0)$ & -2.290 & $(1.325,0.351,0)$ & -2.300 & & \\
\hline & 0.01 & $(1.331,0.334,0)$ & -2.329 & $(1.311,0.324,0)$ & -2.315 & $\left(\frac{17}{9}, \frac{8}{9}, 0\right)$ & -1.210 \\
\hline & 0.001 & $(1.333,0.333,0)$ & -2.333 & $(1.329,0.329,0)$ & -2.333 & & \\
\hline
\end{tabular}

a The optimal solution to the bilevel nonlinear programming.

b The upper level's objective function value of the bilevel nonlinear programming.

For Example 3, following the same procedure as for Example 2, we study the different optimal solutions for varying values of $\epsilon$. Let $z=\left(x_{1}, y_{1}, y_{2}, \lambda_{1}, \lambda_{2}, \lambda_{3}, \lambda_{4}\right)^{T}$ and set the initial point $z^{(0)}=(1,1,1,1,1,1,1)^{T}$. Parameters $\sigma, w^{(0)}, \vartheta^{(0)}, \alpha, \beta$, and $\varepsilon$ are selected as in Example 1. Parameter $\epsilon$ is set to different small values and the results are also presented in Table 1 .

From Table 1, we find that the computed results converge to an optimal solution with decreasing values of $\epsilon$. Moreover, the solutions obtained by the proposed algorithm for the three examples with different values of $\epsilon$, except for the solution in Example 2 for $\epsilon=0.1$, are better than those presented in literature based on a comparison of the objective value for different values of $\epsilon$. Thus we arrive at the conclusion that the proposed algorithm is feasible for solving NBLP problems.

\section{Conclusions}

Bilevel programming problems are intrinsically non-convex and difficult to solve for a global optimum solution. In this paper, we presented an augmented Lagrangian multiplier method to solve NBLP problems. In this algorithm, the KKT condition is used in the lower level problem to transform the NBLP problem into a single nonlinear programming problem with complementary constraints. A CHKS smoothing function is adopted to avoid the difficulty of dealing with a non-differentiable problem because of the complementary condition. An augmented Lagrangian multiplier method is then applied to solve the smoothed nonlinear programming problem to obtain the optimal solution of the NBLP problem. We analyzed the asymptotic properties of the method and derived the condition for solution optimality. Numerical results show that the proposed algorithm is feasible for solving NBLP problems.

\section{Acknowledgements}

This work was supported by the National Natural Science Foundation of China (Nos. 50809004 and 41071323),the State Key Development Program for Basic Research of China (No. 2009CB421104) and the National Major Science and Technology Projects for Water Pollution Control and Management (Nos. 2012ZX07029-002, 2012ZX07203-003).

\section{References}

[1] J. Lu, C.G. Shi, G.Q. Zhang, On bilevel multi-follower decision making: genera framework and solutions, Information Sciences 176 (2006) 1607-1627.

[2] O. Ben-Ayed, C. Blair, Computational difficulties of bilevel linear programming, Operations Research 38 (3) (1990) 556-560.

[3] J.F. Bard, Some properties of the bilevel linear programming, Journal of Optimization Theory and Applications 68 (7) (1991) 146-164.
[4] L. Vicente, G. Savard, J. Judice, Descent approaches for quadratic bilevel programming, Journal of Optimization Theory and Applications 81 (1994) 379-399.

[5] I.C. Herminia, G. Carmen, On linear bilevel problems with multiple objectives at the lower level, Omega 39 (1) (2010) 33-40.

[6] H.S. Shih, U.P. Wen, E.S. Lee, et al., A neural network approach to multiobjective and multilevel programming problems, Computers and Mathematics with Applications 48 (1-2) (2004) 95-108.

[7] S.R. Hejazi, A. Memariani, G. Jahanshahloo, et al., Linear bilevel programming solution by genetic algorithm, Computers and Operations Research 29 (13) (2002) 1913-1925.

[8] G.M. Wang, X.J. Wang, Z.P. Wan, et al., A globally convergent algorithm for a class of bilevel nonlinear programming problem, Applied Mathematics and Computation 188 (1) (2007) 166-172.

[9] G.M. Wang, Z.P. Wan, X.J. Wang, et al., Genetic algorithm based on simplex method for solving linear-quadratic bilevel programming problem, Computers and Mathematics with Applications 56 (2008) 2550-2555.

[10] K.M. Lan, U.P. Wen, H.S. Shih, et al., A hybrid neural network approach to bilevel programming problems, Applied Mathematics Letters 20 (8) (2007) 880-884.

[11] M. Gendreau, P. Marcotte, G. Savard, A hybrid Tabu-ascent algorithm for the linear bilevel programming problem, Journal of Global Optimization 8 (3) (1996) 217-233.

[12] U.P. Wen, A.D. Huang, A simple Tabu Search method to solve the mixedinteger problem bi-level programming problem, European Journal of Operational Research 88 (1996) 563-571.

[13] U.P. Wen, A.D. Huang, A Tabu search approach for solving the linear bilevel programming problem, Journal of the Chinese Institute of Industrial Engineers 13 (1996) 113-119.

[14] H.I. Calvete, C. Gale, M.J. Oliveros, Bilevel model for production-distribution planning solved by using ant colony optimization, Computers and Operations Research 38 (1) (2011) 320-327.

[15] R.J. Kuo, C.C. Huang, Application of particle swarm optimization algorithm for solving bi-level linear programming problem, Computers and Mathematics with Applications 58 (2009) 678-685.

[16] R.J. Kuo, Y.S. Han, A hybrid of genetic algorithm and particle swarm optimization for solving bi-level linear programming problem? A case study on supply chain model, Applied Mathematical Modelling 35 (2011) 3905-3917.

[17] Z.P. Wan, G.M. Wang, B. Sun, A hybrid intelligent algorithm by combining particle swarm optimization with chaos searching technique for solving nonlinear bilevel programming problems, Swarm and Evolutionary Computation 8 (2013) 26-32.

[18] B. Chen, P.T. Harker, A non-interior point continuation method for linear complementarity problems, SIAM Journal on Matrix Analysis and Applications 14 (1993) 1168-1190.

[19] C. Chen, O.L. Mangasarian, Smoothing methods for convex inequalities and linear complementarity problems, Mathematical Programming 71 (1995) 5169.

[20] X.J. Chen, Smoothing methods for complementarity problems and their applications: a survey, Journal Operations Research Society of Japan 43 (2000) 32-47.

[21] F. Facchinei, H. Jiang, L. Qi, A smoothing method for mathematical programming with equilibrium constraints, Mathematical Programming 85 (1999) 107-134.

[22] S. Dempe, Foundation of Bilevel Programming, Kluwer Academic Publishers, London, 2002.

[23] G.M. Wang, K.J. Zhu, Z.P. Wan, An approximate programming method based on the simplex method for bilevel programming problem, Computers and Mathematics with Applications 59 (2010) 3355-3360.

[24] J.B.E. Etoa, Solving quadratic convex bilevel programming problems using a smoothing method, Applied Mathematics and Computation 217 (2011) 66806690.

[25] Z.H. Gümüs, C.A. Floudas, A shuffled global optimization of nonlinear bilevel programming problems, Journal of Global Optimization 20 (2001) 1-21. 
[26] Y. Chen, M. Florian, The nonlinear bilevel programming problem: formulations, regularity and optimality conditions, Optimization 32 (1995) 193-209.

[27] H. Scheel, S. Scholtes, Mathematical programs with complementarity constraints: stationarity, optimality, and sensitivity, Mathematics of Operations Research 25 (1995) 1-22.

[28] M. Fukushima, J.S. Pang, Convergence of a smoothing continuation method for mathematical problem with complementarity constraints, Lecture Notes in Economics and Mathematical Systems 477 (1999) 105-116.

[29] Y. Tao, A class of smoothing methods for mathematical programs with complementarity constraints, Applied Mathematics and Computation 186 (2007) 1-9.
[30] M.R. Hestenes, Multiplier and gradient methods, Journal of Optimization Theory and Applications 4 (1969) 303-320.

[31] M.J.D. Powell, A method for nonlinear constraints in minimization problems, in: R. Fletcher (Ed.), Optimization, Academic Press, New York, 1969, pp. $283-$ 298.

[32] M.A. Amouzegar, A global optimization method for nonlinear bilevel programming problems, IEEE Transaction on Systems, Man, and Cybernetics, Part B (Cybernetics) 29 (6) (1999) 771-777.

[33] W. Zhong, N. Xu, Boltzmann machine method for two-level decision making, Journal of Systems Engineering 10 (1) (1995) 7-13.

[34] J. Bard, Practical Bilevel Optimization: Algorithm and Applications, Kluwer Academic Publishers, Dordrecht, 1999. 